University of South Carolina

Scholar Commons

\title{
Application of the Empirical Mode Decomposition and Hilbert- Huang Transform to Seismic Reflection Data
}

\author{
Bradley M. Battista \\ Camelia C. Knapp \\ University of South Carolina - Columbia, camelia@geol.sc.edu \\ Tom McGee \\ University of Mississippi Main Campus \\ Vaughn Goebel
}

Follow this and additional works at: https://scholarcommons.sc.edu/geol_facpub

Part of the Earth Sciences Commons

\section{Publication Info}

Published in Geophysics, Volume 72, Issue 2, 2007, pages H29-H37.

Battista, B. M., Knapp, C. C., McGee, T., \& Goebel, V. (2007). Application of the empirical mode decomposition and Hilbert-Huang transform to seismic reflection data. Geophysics, 72 (2), H29-H37. (C) Geophysics 2007, Society of Exploration Geophysicists 


\title{
Application of the empirical mode decomposition and Hilbert-Huang transform to seismic reflection data
}

\author{
Bradley Matthew Battista ${ }^{1}$, Camelia Knapp ${ }^{1}$, Tom $\mathrm{McGee}^{2}$, and Vaughn Goebel ${ }^{3}$
}

\begin{abstract}
Advancements in signal processing may allow for improved imaging and analysis of complex geologic targets found in seismic reflection data. A recent contribution to signal processing is the empirical mode decomposition (EMD) which combines with the Hilbert transform as the HilbertHuang transform (HHT). The EMD empirically reduces a time series to several subsignals, each of which is input to the same time-frequency environment via the Hilbert transform. The HHT allows for signals describing stochastic or astochastic processes to be analyzed using instantaneous attributes in the time-frequency domain. The HHT is applied herein to seismic reflection data to: (1) assess the ability of the EMD and HHT to quantify meaningful geologic information in the time and time-frequency domains, and (2) use instantaneous attributes to develop superior filters for improving the signal-to-noise ratio. The objective of this work is to determine whether the HHT allows for empirically-derived characteristics to be used in filter design and application, resulting in better filter performance and enhanced signal-to-noise ratio. Two data sets are used to show successful application of the EMD and HHT to seismic reflection data processing. Nonlinear cable strum is removed from one data set while the other is used to show how the HHT compares to and outperforms Fourier-based processing under certain conditions.
\end{abstract}

\section{INTRODUCTION}

This work is an introduction to the application of the empirical mode decomposition (EMD) and related Hilbert-Huang transform (HHT) (Huang et al., 1998) to seismic reflection data. The application of the EMD and HHT to these types of data has yet to be recog- nized as a standard application by the exploration seismology community. Therefore, it is the purpose of this work to demonstrate the ability for these new techniques, recently accepted in other disciplines, to improve seismic reflection data quality. This work does not attempt to replace conventional seismic processing methods, but instead, to complement them with the addition of the EMD and HHT. In this respect, the EMD is proposed as a highly effective time-domain filter. Additionally, the HHT is compared to the Fourier transform to the extent that a signal may be characterized with respect to time, frequency, or both simultaneously. The only significant difference is that the HHT can derive instantaneous amplitude, phase, and frequency for a signal with no a priori knowledge; a priori referring to sample frequency and local disruptions of a time series such as noise spikes and step functions (Huang et al., 1998).

\section{Empirical mode decomposition}

The EMD is designed to reduce nonstationary, multicomponent signals to a series of amplitude- and frequency-modulation (AMFM) contributions. The EMD, since its formal introduction by Huang et al. (1998), has yet to be developed into a formal algorithm. This stems from the empirical nature of the process. However, many have written codes to perform the EMD. Among them, and the starting point for this work, are the codes of Rilling et al. (2002). Generally speaking, the overall result of the decomposition is to successively remove the highest frequencies from a signal. The net result is to create a bank of subsignals, termed intrinsic mode functions (IMF), whose sum produces the original signal. The last IMF or residual is of the lowest order. In this manner, Rilling et al. (2004b) have described the EMD to behave as a dyadic filter bank under various conditions. This can also be thought of as having several filters of overlapping frequency content. Therefore, the EMD can be used to gain significant information inherent to the signal. Further, the EMD differs from wavelet decomposition in that the filters of the filter bank do not correspond to sub-band filtering but instead to signal-

Peer-reviewed code related to this article can be found at http://software.seg.org/2007/0003. Manuscript received by the Editor November 11, 2005; revised manuscript received September 29, 2006; published online March 2, 2007.

${ }^{1}$ University of South Carolina, Department of Geological Sciences, Columbia, South Carolina. E-mail: bbattista@geol.sc.edu; camelia@geol.sc.edu.

${ }^{2}$ University of Mississippi, CMRET, University, Misssissippi.E-mail: tmm @ olemiss.edu.

${ }^{3}$ Lookout Geophysical Company, Palisade, Colorado. E-mail: vgoebel@ aol.com.

(C) 2007 Society of Exploration Geophysicists. All rights reserved. 
dependent, time-variant filters. Flandrin and Gonçalvès (2004) elaborate on the differences and similarities of EMD and wavelet decomposition.

The EMD has received much focus in the signal processing community, but has been given little attention by the exploration seismology community. Magrin-Chagnolleau and Baraniuk (1999) demonstrated that seismic traces could be analyzed using EMD. Additionally, similarities in the types of signals being analyzed allowed for the works of Rilling et al. (2004a, b) to be considered. Together, these works analyze the nature and use of the EMD for detrending and denoising data - a common necessity in the seismic exploration industry. Therefore, the foundation is set for further investigation of the potential for EMD and HHT to optimally analyze seismic reflection data.

\section{Hilbert-Huang transform}

In seismic signal processing, the Hilbert transform is commonly used to generate a complex time series or analytic signal. The benefit is that instantaneous attributes can be derived from complex traces. However, accurate and meaningful computation of these attributes requires that the input signal's start and end have zero amplitude, and it contains no trend that introduces a nonzero mean (Dix, 1949). In this regard, perhaps the most significant seismic use for the EMD is to prepare a signal for input to the Hilbert transform. Together, the EMD and the Hilbert transform are labeled the Hilbert-Huang transform. Huang et al. (1998) explain how all of the IMFs may be passed into a common time-frequency domain where the instantaneous amplitude and phase can be analyzed with high resolution. The benefit of preceding the Hilbert transform with the EMD is that signals exhibiting stochastic behavior may also be transformed to yield meaningful physical information in the time-frequency domain. Meaningful physical information refers to instantaneous attributes that are free of artificial harmonics and/or irregular phase caused by a nonzero mean. Furthermore, analyzing instantaneous attributes in the time-frequency domain provides the most comprehensive environment for filter design because both time and frequency domains are considered.

\section{CUSTOMIZATION AND DESIGN}

\section{Empirical mode decomposition}

The objective of the EMD is to empirically separate a signal into several subsignals of varying, and possibly overlapping, frequency content. Each of the subsignals is referred to as an intrinsic mode function because it is empirically derived from the data (i.e., there are no user-specified filters). The EMD produces a bank of IMFs whose sum yields the original signal. The first IMFs produced contain the highest frequency components of a signal while the latter contain the lowest frequency components. Two questions are presented and answered to clarify how the EMD works. First, what is an IMF? Second, how is an IMF computed? The answer to the first question is simple. The total number of extrema (peaks and troughs) in an IMF must not differ from the total number of zero crossings by more than one, and the IMF must not contain a nonzero mean (Huang et al., 1998). The method of producing IMFs, and the answer to the second question, lies in the concept referred to by Huang et al. (1998) as sifting.
The result of sifting is to remove all trends preventing a subsignal from fitting the criteria of an IMF. A signal that already fits the criteria for an IMF will produce no further IMFs during the EMD. Otherwise, the signal's extrema are found and separated into peaks and troughs. A cubic spline is fit to each separately. This envelopes the signal within two curve fits, one that rides along the peaks and one that rides along the troughs. The average of the two cubic-spline fits is taken as a function of time, and is referred to as the mean spline, because it visually resembles a low-order, running-average curve fit. The mean spline is subtracted to produce a new signal. The process is repeated on each new signal until the criteria for an IMF are met. It is nearly impossible to achieve a mean spline that is exactly zero for the signal's duration, so a final stopping criterion must be set to determine when sifting has effectively produced an IMF. This criterion is a predetermined rms tolerance between two consecutive components of sifting. Let $s_{0}(t)$ be sifted once to yield its first component, $s_{1}(t)$, by removing its average spline, $m_{0}(t)$. The rms is determined for each $s_{0}(t)$ and $s_{1}(t)$, and their difference is compared to the predetermined tolerance. Sifting continues according to equation 1 if the difference between rms values is greater than the tolerance.

$$
\begin{gathered}
\frac{1}{J}\left(\left(\sum_{j=1}^{J} s_{n-1}(t)^{2}\right)^{\frac{1}{2}}-\left(\sum_{j=1}^{J} s_{n}(t)^{2}\right)^{\frac{1}{2}}\right) \\
>\text { tolerance, } \\
s_{n+1}(t)=s_{n}(t)-m_{n}(t),
\end{gathered}
$$

where $n=1,2,3, \ldots$ and $J$ is the number of samples in the time series. The first IMF, having had several lower frequency components removed, contains only the highest frequency components of the signal that do not cause it to violate the IMF criteria. Subtracting the first IMF from the original signal produces a new signal which may be sifted to form a second IMF. Sifting and production of IMFs continues until the process is manually terminated or the remaining residual contains at most three extrema. Therefore, IMFs can be related to a signal in accordance with equation 2 ,

$$
c_{0}(t)=\left(\sum_{n=1}^{N} c_{n}(t)\right)+r(t)
$$

where $c_{0}(t)$ is the original signal, $c_{n}(t)$ are IMFs, $r(t)$ is a residual, if any, and $N$ is the total number of IMFs. Figure 1 illustrates the EMD of a signal composed of three pure tones.

\section{Instantaneous attributes}

Let $X_{n}(t)$ be a bank of IMFs and $Y_{n}(t)$ be a bank of their Hilbert transforms. A bank of analytic signals, $Z_{n}(t)$, can be determined as

$$
Z_{n}(t)=X_{n}(t)+i Y_{n}(t)
$$

where $n$ denotes the IMF in the bank. The bank of analytic signals affords the ability to determine instantaneous amplitude and phase in the time domain. Equations 4 and 5 show how this is done: 


$$
\begin{aligned}
& a_{n}(t)=\sqrt{X_{n}(t)^{2}+Y_{n}(t)^{2}}, \\
& \phi_{n}(t)=\tan ^{-1}\left(\frac{Y_{n}(t)}{X_{n}(t)}\right) .
\end{aligned}
$$

Amplitude, $a_{n}(t)$, and phase, $\phi_{n}(t)$, for each IMF in the bank are found using simple trigonometry. Instantaneous frequency is determined within the intervals between samples and not at individual samples. This stems from the fact that frequency, $f_{n}(t)$, is the first derivative of phase according to equation 6

$$
f_{n}(t)=\frac{1}{2 \pi} \frac{d \phi_{n}}{d t}
$$

Once determined, amplitude, phase, and frequency can be time-sorted and displayed in a time-frequency fashion. This produces a spectrogram at the highest possible resolution; that is, for every sample in time there is a corresponding amplitude, phase, and frequency. Additionally, the temporal summation of amplitude, $a_{(f, t)}$, with respect to frequency shown in equation 7 produces a marginal spectrum, $h_{f}$, in similar manner to that of a periodogram:

$$
h_{f}=\int_{0}^{T} a_{(f, t)} d t .
$$

The difference is that there is no spectral windowing as with the periodogram because of the use of instantaneous attributes, and there may exist frequencies having no energy. However, this does not happen with broadband signals, and the result resembles a power spectrum. Figure 2 provides the time-frequency results of determining the Hilbert-Huang transform on the IMFs derived for the three-component signal from Figure 1. Validation of the instantaneous characteristics found in this manner is determined in much the same way as with the Fourier transform. Equation $8 \mathrm{em}-$ ploys Euler's relationship to reconstruct a time series from its instantaneous amplitude and phase. The real part $(\mathfrak{R})$ of the summation is equal to the original time series.

$$
X(t)=\mathfrak{R} \sum_{n=1}^{N} a_{n} e^{i \phi_{n} t} .
$$

\section{Data analysis}

The empirical nature of the EMD causes it to be sensitive to both analog and digital characteristics of a signal. An analog example masks a high-frequency signal with strong, low-frequency trends. A digital example would be quantizing or aliasing the data with improper sampling rates. Adequate sampling for the EMD should be much higher than that determined from conventional sampling theory. As explained in Appendix A,
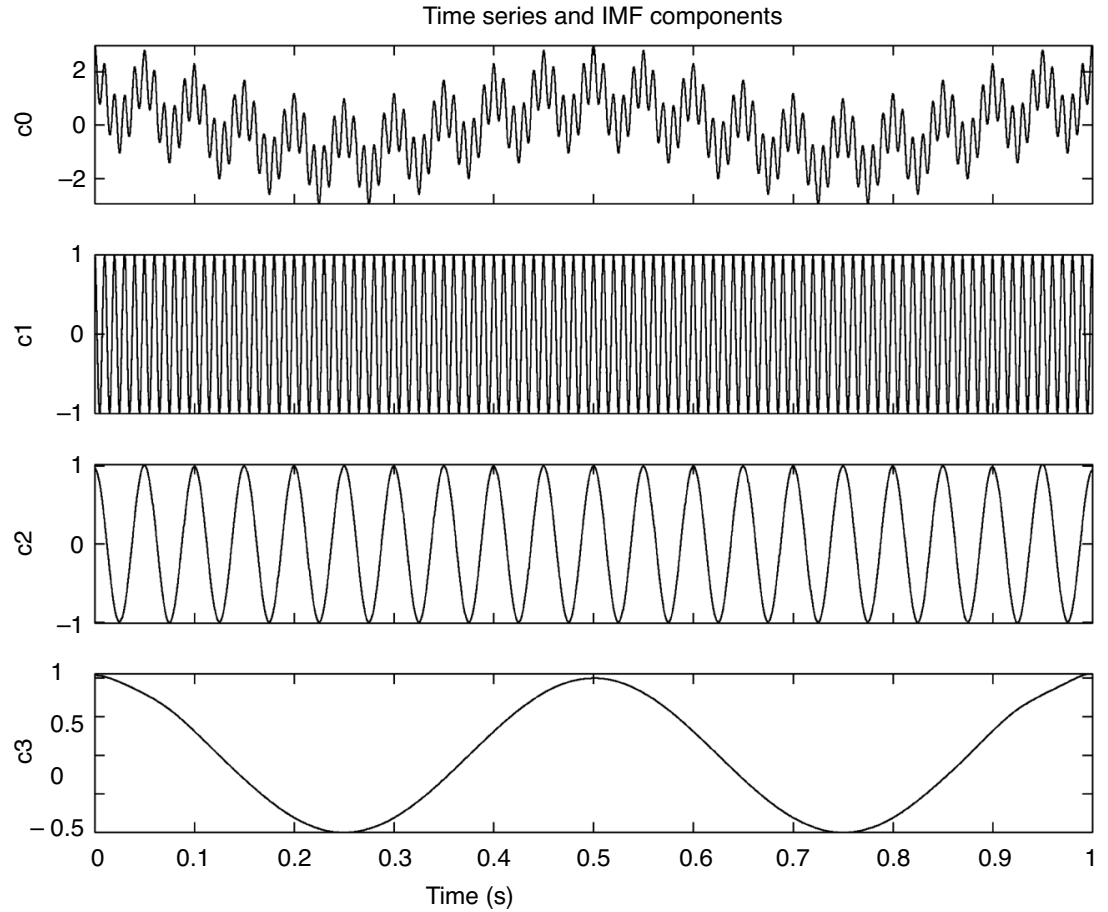

Figure 1. A signal, $c_{0}(t)$, composed of three frequencies $(2,20$, and $100 \mathrm{~Hz})$ is empirically decomposed to each of the principal frequencies $\left(c_{1}(t)-c_{3}(t)\right)$.

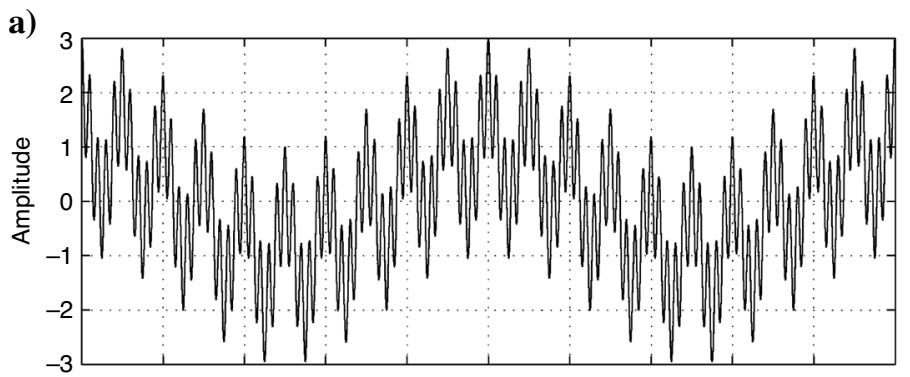

b)

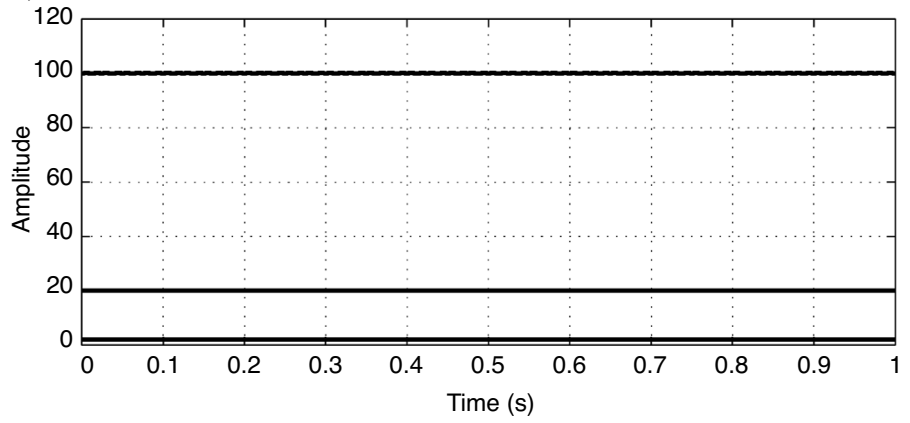

c)

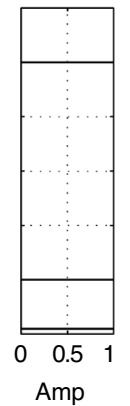

Figure 2. Hilbert-Huang transform of a signal composed of three frequencies $-2,20$, and $100 \mathrm{~Hz}$. The time series in (a) is transformed to the time-frequency domain in (b). The Hilbert marginal spectrum shown in (c) is produced as the temporal sum of amplitudes for every frequency in (b). 
the discrepancy stems from the invalid assumption that finite-length pulses, such as seismic pulses, are band limited (Shannon, 1949; Slepian, 1976).

\section{EMD as a time-domain filter}

Two data sets are used to analyze the performance of the EMD and HHT with respect to seismic reflection data. The first comes from a single-channel, shallow source/deep receiver (SSDR) data set from the Gulf of Mexico. These data carry strong, low-frequency energy generated from the strumming of the receiver cable as the receiver was towed through the water column. Cable strum is subject to nonlinear forcing, and presents a unique opportunity for the EMD to demonstrate its strength as a time-domain filter. The cable-strum noise is removed using only the EMD as a time-domain filter by generating several narrow-band IMFs and summing those that do not contain the low-frequency noise. The IMFs containing noise may be manually removed after visual inspection or handled by way of a user-defined crosscorrelation to the original trace.

\section{HHT as a time-frequency filter}

The second data set comes from a high-quality, multichannel seismic survey from the Caspian Sea. There is very little noise in these data and there exists a strong bottom-simulating reflector from Diaconescu et al. (2001). There is no significant need to use the EMD as a time-domain filter as with the first data set because the signal-to- noise ratio is already very high. The EMD is applied to these data only as a prerequisite to the HHT. The HHT is first used to compare filtering in the time-frequency domain versus that of the frequency domain using the traditional Fourier transform. Secondly, the instantaneous attributes derived from the HHT are compared to those produced by the Hilbert transform without the use of the EMD. These exercises demonstrate both the strength of time-frequency domain filtering and the necessity of using the EMD with the Hilbert transform.

\section{OPTIMIZATION AND FILTER COMPARISON}

\section{Parameter optimization for the EMD}

The EMD is very sensitive to both the physical and digital characteristics of the signal being analyzed. The sensitivity is high enough to pick up quantization and fidelity errors. Quantization errors result from rounding errors introduced when a continuous function is sampled at discrete locations (Oppenheim and Schafer, 1989). Loss of fidelity allows for amplitude modulations even when sampling faster than the conventional Nyquist frequency. Most signal processing techniques for seismic data are not significantly affected by this.

Figure 3 shows that the IMFs from a pure $60 \mathrm{~Hz}$ tone sampled every $1000 \mu \mathrm{sec}(1 \mathrm{kHz})$ introduce a $20 \mathrm{~Hz}$ tone. The same signal sampled every $333 \mu \mathrm{sec}(\approx 3 \mathrm{kHz})$ does not produce the $20 \mathrm{~Hz}$ tone after EMD because the fidelity error has been significantly reduced. Furthermore, low-energy dots in Figure 3 result from edge effects in the Hilbert transform. These edge effects are unavoidable in the case of a pure cosine function because it is impossible for it to meet Dix's (1949) criteria of zero-amplitude beginning and end with zero mean throughout. However, following Slepian (1976), these points can be ignored because they fall below the level of significant energy. Moreover, loss of fidelity is inevitable for any seismic pulse, and the choice of sample rate should be the highest possible if the assumption is held that seismic pulses possess infinite bandwidth, Appendix A defends this assumption. This lends support to McGee (2000), who suggested seismic signals should be sampled as fast as possible to respect the Heisenberg Uncertainty Principle, stating that time-limited signals have infinite bandwidth and band-limited signals have no beginning or end.

The EMD sometimes exhibits difficulty separating weak trends from strong trends. This mostly occurs when a signal contains strong, low-frequency components with weak, high-frequency components riding along. The sifting process of the EMD may miss detect the weak components when determining the signal's envelope and mean. To summarize sifting, a signal's extrema are located and separated into upper (peaks) and lower (troughs). A cubic spline is drawn through each and they are averaged to form the mean spline. The mean spline can significantly stray from the signal in cases dealing with strong trends. For example, parts of the mean spline could lie outside of the signal's amplitude enve-

Figure 3. Effect of sample interval on the EMD. (a) Hilbert marginal spectrum for a $60 \mathrm{~Hz}$ tone before performing the EMD. (b) Hilbert marginal spectrum for all IMFs produced by the EMD for a $60 \mathrm{~Hz}$ tone with a $1000 \mu$ sec sample interval. The introduction of a weak, $20 \mathrm{~Hz}$ peak results from slight amplitude modulation caused by the EMD's sensitivity to fidelity. (c) Hilbert marginal spectrum for all IMFs produced by the EMD for a $60 \mathrm{~Hz}$ tone with a $333 \mu$ sec sample interval. 
lope. Artificial trends are produced during sifting when the poorly determined mean splines are subtracted from the data. Figure 4 shows both the nature of this problem as well as a working solution. The problem is that strong, short-lived trends prohibit the detection of extrema because the weaker signal riding on the trend cannot express a change of slope (i.e., peaks and troughs are lost in the trend). The "missing" extrema can be located by analyzing the distribution of extrema for the whole signal. Extrema spacing is a measure of the dominant frequencies in the signal, and a large gap in extrema suggests the presence of a strong trend. Thus, a strong, local trend is defined as a portion of the signal where the extrema spacing is greater than two standard deviations from the mean extrema spacing. These portions of signal are fitted with a third-order polynomial that is subtracted before determining extrema. The result, as shown in Figure 4 , is to constrain the mean spline such that it cannot separate from the signal. Further, the introduction of false trends is significantly minimized by using well-constrained mean splines. An additional benefit of this is to produce more IMFs of narrower bandwidth than before. Results of using the EMD with and without this optimization are shown in the following section. Furthermore, this modification can be considered permanent, and does not need to be adjusted for other data sets. It is presented here to demonstrate how the EMD is adapted for use with seismic signals.

\section{Cable strum removal using the EMD}

The EMD is applied to a single-channel seismic reflection profile taken in the Gulf of Mexico using a shallow source with a deeptowed receiver. There is a significant amount of noise contributed to the receiver because of cable strum as shown in Figure 5. The energy of the cable strum far exceeds that of the seismic reflections from the seafloor and below. However, performing the EMD, even under poor conditions, helps to bring out the desired data. Figure 6 shows the same traces after performing EMD and filtering without any parameter optimization as described above. Most of the cable strum has been removed but there is obvious evidence for the introduction of artificial trends; these being the high amplitude noise in Figure 6. Parameter optimization of the EMD reduces the introduction of artificial trends almost completely and produces IMFs of narrow bandwidth. Therefore, it is much easier to separate the cable strum in the time domain by summing IMFs without cable strum. In this manner, the EMD is used as a signal-dependent, time-variant filter. Figure 7, having had EMD parameterization to reduce artificial trends, presents the ideal results. Most of the traces contain the same number of IMFs but some contain less. The ability to decide which IMFs are most representative is subject to interpretation, but can be guided. It was decided that any IMFs containing cable strum and not containing energy for the seafloor reflection could be removed based on the idea that the seafloor horizon should reflect the most energy and attenuate the least. This criterion proves highly effective, as seen in Figure 7. The data in the upper panel is not present in the filtered noise in the lower panel.

b) spline.

\section{Time-frequency versus frequency-domain filtering}

The HHT is used to derive instantaneous phase and amplitude for a signal. Instantaneous frequency can be derived from phase as described previously in equation 6 . In this manner, a time-frequency representation is achieved wherein every sample is accounted for. The Hilbert transform can be approximated via forward and inverse discrete Fourier transform only if the signal satisfies the amplitude and mean criteria described by Dix (1949), which are optimally achieved using the EMD. Additionally, the HHT and Fourier transform can apply the same transfer function to the data with nearly identical results. Figure 8 illustrates the results of applying the same frequency response, a sixth-order Butterworth bandpass filter with a passband of $100-300 \mathrm{~Hz}$, to the same data set. Figure 8a shows the filtered data, while Figure $8 \mathrm{~b}$ shows the traditional method using FFT. Clearly, the two methods of filtering are related as the results are nearly the same. However, upon closer look, there are differences. These differences can be caused by two major factors. First, the HHT may produce invalid attributes, if any of the employed IMFs carry a trend which they should not if the EMD is properly performed; meaning it was prevented from sifting completely. Second, a zero-phase filter is achieved via Fourier transform by filtering both the forward and reverse directions, which effectively applies the filter twice. Therefore, the HHT bandpass filter maintains a higher spectral resolution. Even so, the two forms of filtering converge on the same result when optimum detrending has occurred prior to filtering. Further, neither application outperforms the time-domain implementation of the EMD as seen in Figure 7. Filter comparison is also performed on high-quality data to show how robust the HHT really is. We noted above that applying a bandpass filter using FFT and HHT produced nearly the same result. Figure 9 reemphasizes this point by applying a bandpass filter with a passband of $15-60 \mathrm{~Hz}$ to data from the Caspian Sea containing a buried gas hydrate (Diaconescu et al., 2001). Notice there are many similarities between the
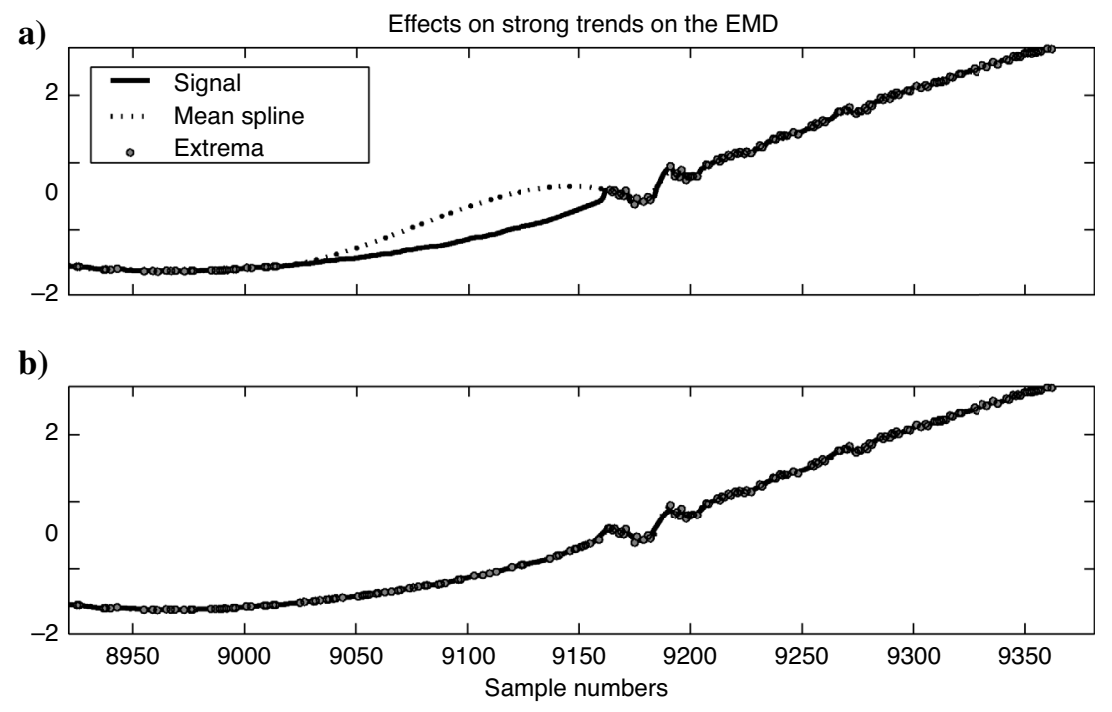

Figure 4. Effects of strong trends on the EMD. (a) Example of how the mean spline can stray from a signal in the presence of a strong trend caused by lack of extrema. (b) Results of an automated routine that finds the missing extrema to properly constrain the mean 


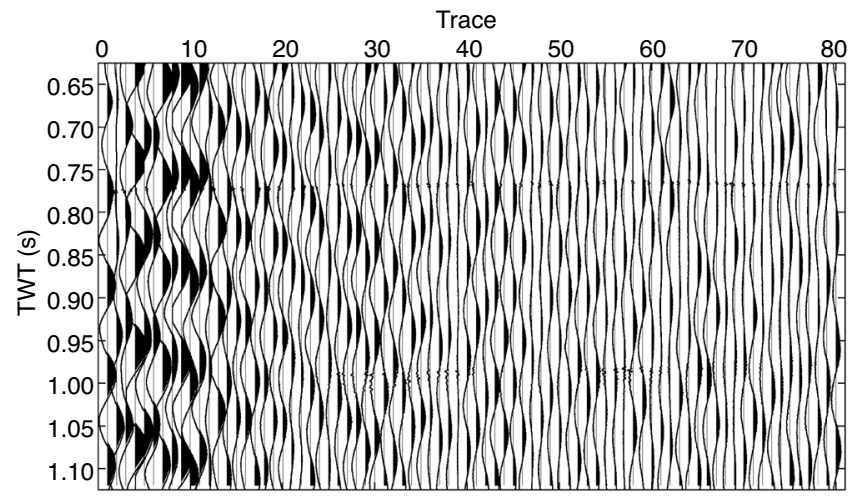

Figure 5. Single-channel seismic reflection data containing strong, low-frequency cable strum. All of the horizontal trends are data from the seafloor and below. They are much weaker than the trends they ride along. The horizontal trend near $0.77 \mathrm{~s}$ is the seafloor. Later, these data are separated from the cable strum.

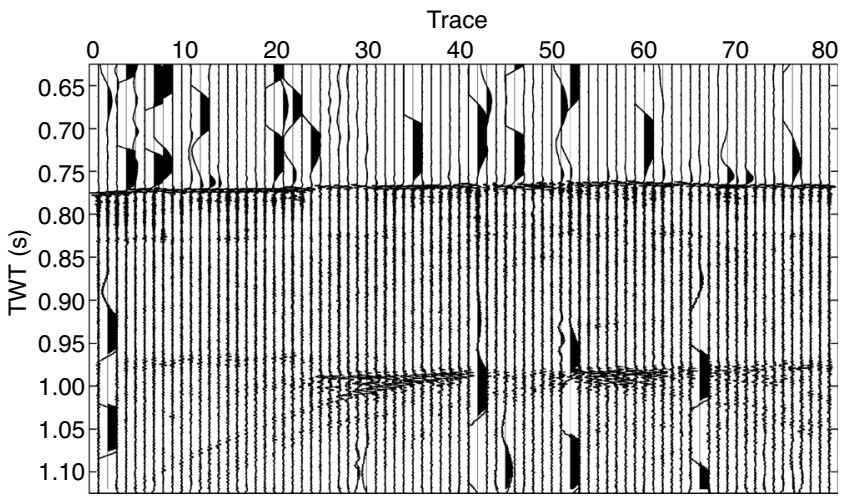

Figure 6. Primary results of the EMD attempting to remove cable strum from seismic reflection data. Strong, low-frequency noise remains. This noise is both cable strum and artificial noise caused by improper sifting in the presence of strong trends.
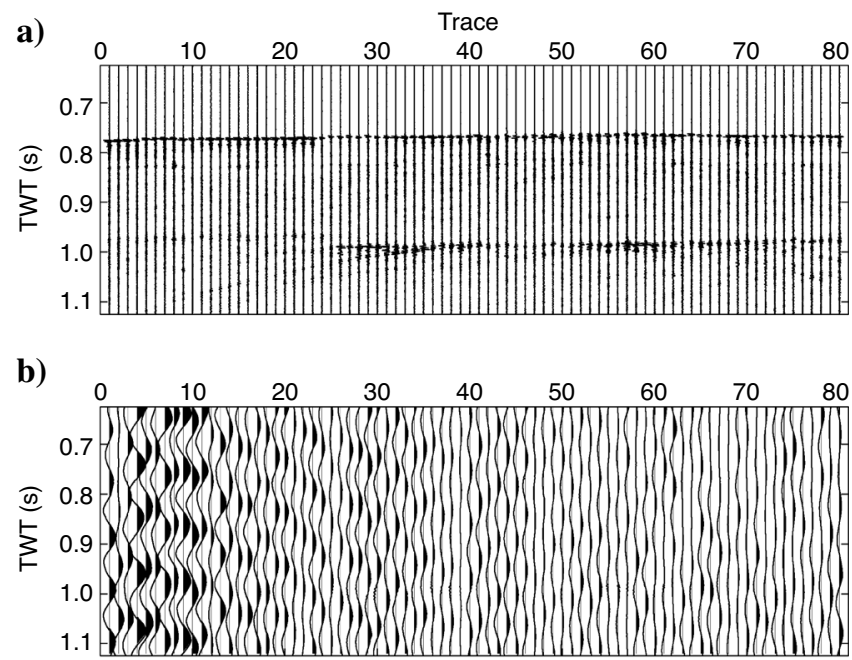

Figure 7. Final results of the EMD attempting to remove cable strum from seismic reflection data. The data in (a) has been empirically separated from the noise in (b). Statistical evaluation of extrema spacing and partial sifting during the EMD has allowed for improved separation of cable strum.
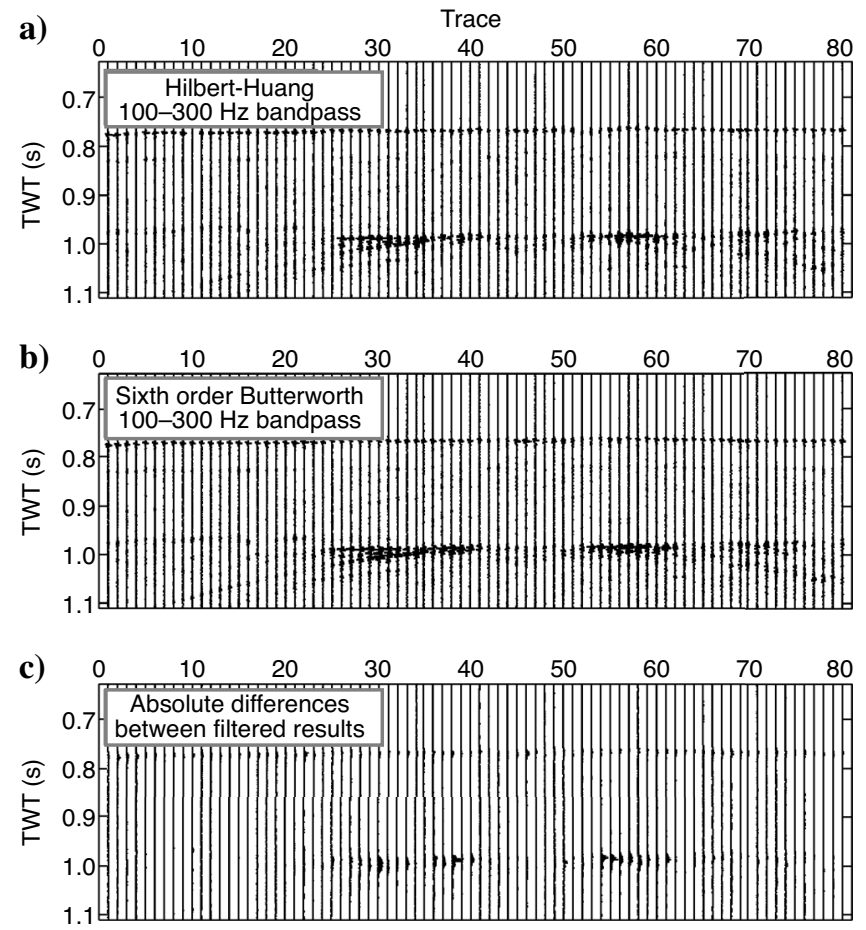

Figure 8. Comparison of a zero-phase, sixth-order Butterworth filter applied using FFT and HHT. (a) Data filtered using instantaneous attributes from HHT. (b) Data filtered using traditional convolution. (c) Residual between (a) and (b) above. Notice how both techniques effectively remove cable strum and portions of signal.

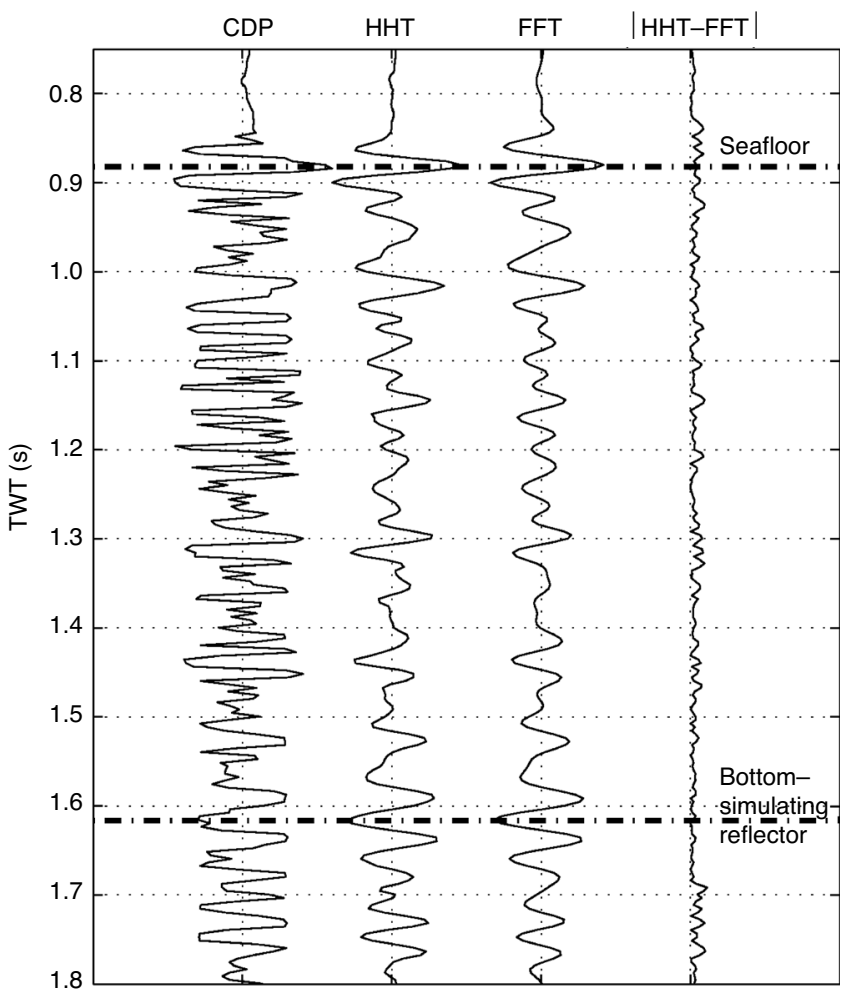

Figure 9. Comparison of a zero-phase, sixth-order Butterworth filter applied to a CDP trace using FFT and HHT. Notice the phase difference and additional smoothing of the FFT filter not present in the HHT filter. The residual between the two filters, |HHT-FFT|, in the last trace further demonstrates this point. 
two techniques. However, as stated before, the HHT posseses higher resolution and respects the phase of the original signal better than the FFT-based filter.

\section{HHT versus Hilbert transform}

The HHT differs not only from Fourier-based techniques but also from a standard Hilbert transform. As a reminder, the only algorithmic difference between the HHT and the Hilbert transform is the use of the EMD to prepare the signal. Figure 10 illustrates the application of EMD to a CDP gather, post normal-moveout removal, from the Caspian Sea data set. Figure 10a shows the individual IMFs, where Figure 10b shows the cumulative sum of the IMFs for each CDP. The HHT is applied to the gather by applying the Hilbert transform to the IMFs and not the original CDP traces. The strength of the HHT becomes quite apparent when comparing the attributes derived from the HHT versus the Hilbert transform. Figure 11 presents a time-frequency spectrogram, (Figure 11b), and Hilbert marginal spectrum, (Figure 11c), for the CDP trace (Figure 11a). It appears as though the signal is composed of broadband energy as the instantaneous frequencies are widely distributed. In contrast, Figure 12 suggests the signal is primarily composed of stable, low-frequency components easily seen as trends. The broadband energy components are still present. The differences occur because the CDP trace carries localized DC shifts while its IMFs do not, and the Hilbert transform breaks down in the presence of a nonzero mean. Both sets of attributes, from HHT and Hilbert transform, reproduce the original trace after the inverse transform is applied, although the HHT representation provides optimum results in the time-frequency domain. ior. The HHT requires no knowledge of the signal's sample rate or frequency content because the EMD empirically reduces data to intrinsic modes. In addition to this, the HHT is effectively a time-domain operation, and can perform zero-phase filtering with a single operation unlike its Fourier counterpart. Furthermore, HHT-based filtering retains greater spectral content than Fourier-based filtering. Zero-phase Fourier-based filters are typically achieved in two steps,
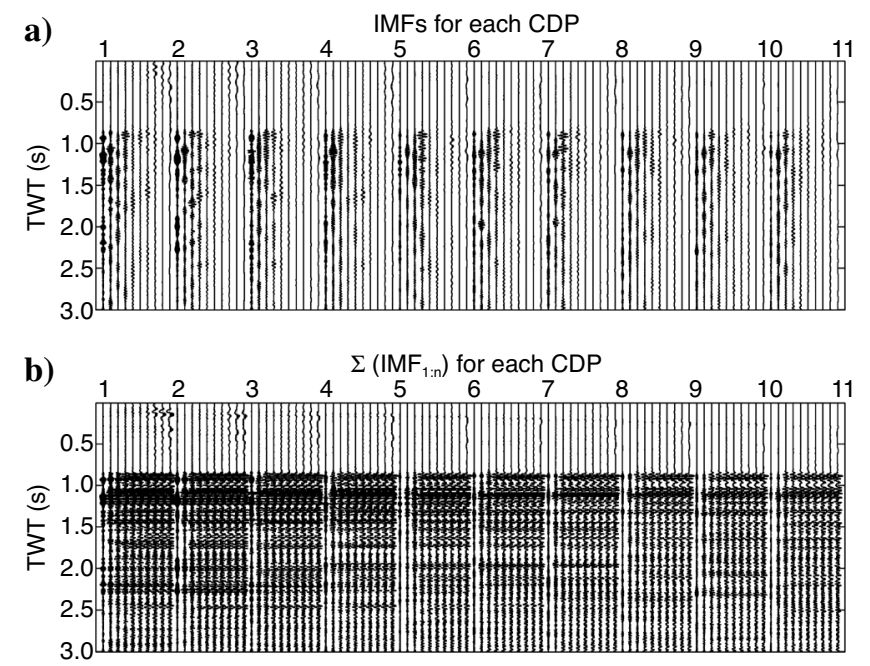

Figure 10. EMD of a CDP gather. (a) Filter bank containing each of the 10 IMFs for each CDP. (b) Cumulative sums of the IMFs in the filter bank, which yield the original signal in the last trace for each CDP.

\section{DISCUSSION}

The objectives of this work are determine the ability of the EMD and HHT in order to quantify meaningful geologic information in the time and time-frequency domains and to develop enhanced filters in the time-frequency domain using instantaneous attributes to improve signal-tonoise ratio. The results presented herein suggest that both of these objectives are met. The integrity of the EMD is crucial to the ability of the HHT to outperform traditional Fourier-based techniques. Therefore, parameterization of the EMD to a given type of data proves to be the highest priority for the application of HHT to seismic reflection data processing. EMD parameterization allows for both the production of narrow-band IMFs as well as the most detailed calculation of instantaneous phase, amplitude, and frequency. Additionally, designing filters around these attributes produces results nearly identical to those produced by standard Fourier-based filters. However, the tolerance for poorly behaved signals, such as those of the cable-strum example, is more readily accepted by the HHT than with Fourier-based techniques.

Several characteristics of the HHT set it apart from other filtering methods, in addition to the acceptance of signals exhibiting stochastic behav-

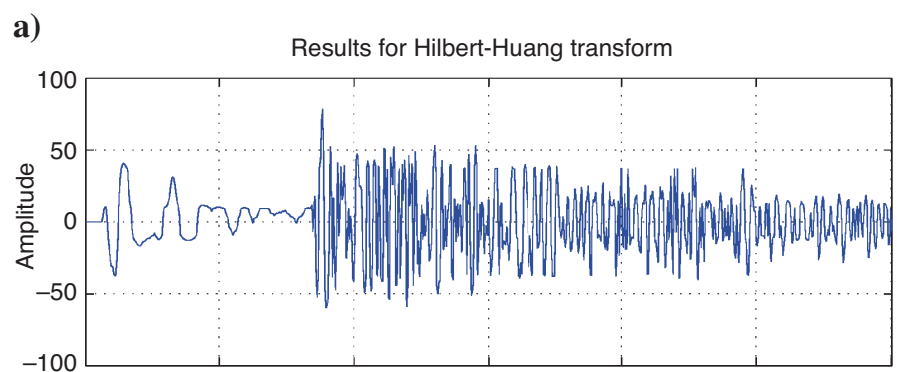

b)

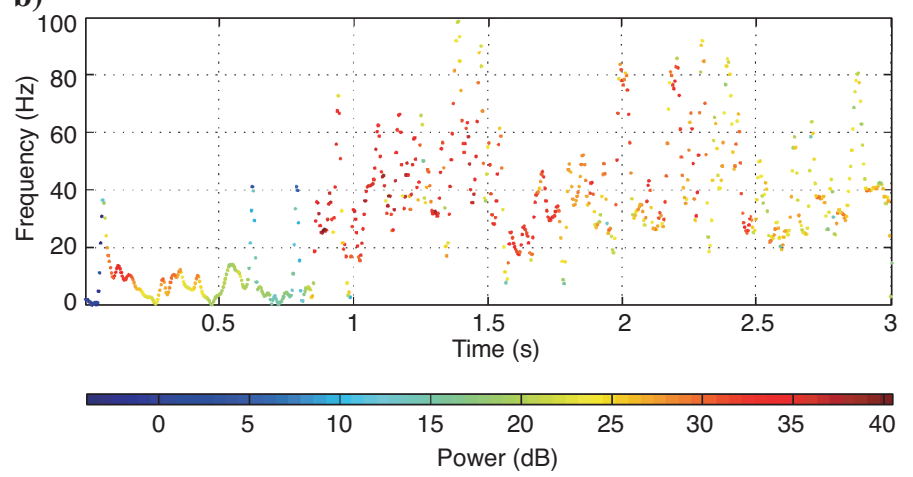

c)

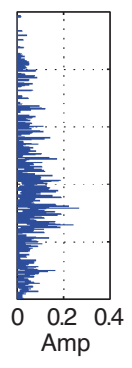

Figure 11. Hilbert transform of a stacked CDP gather having no EMD applied. (a) The time series analyzed. (b) Signal power plotted in time-frequency. (c) Hilbert marginal spectrum created as a temporal sum of instantaneous amplitude for given frequencies. 

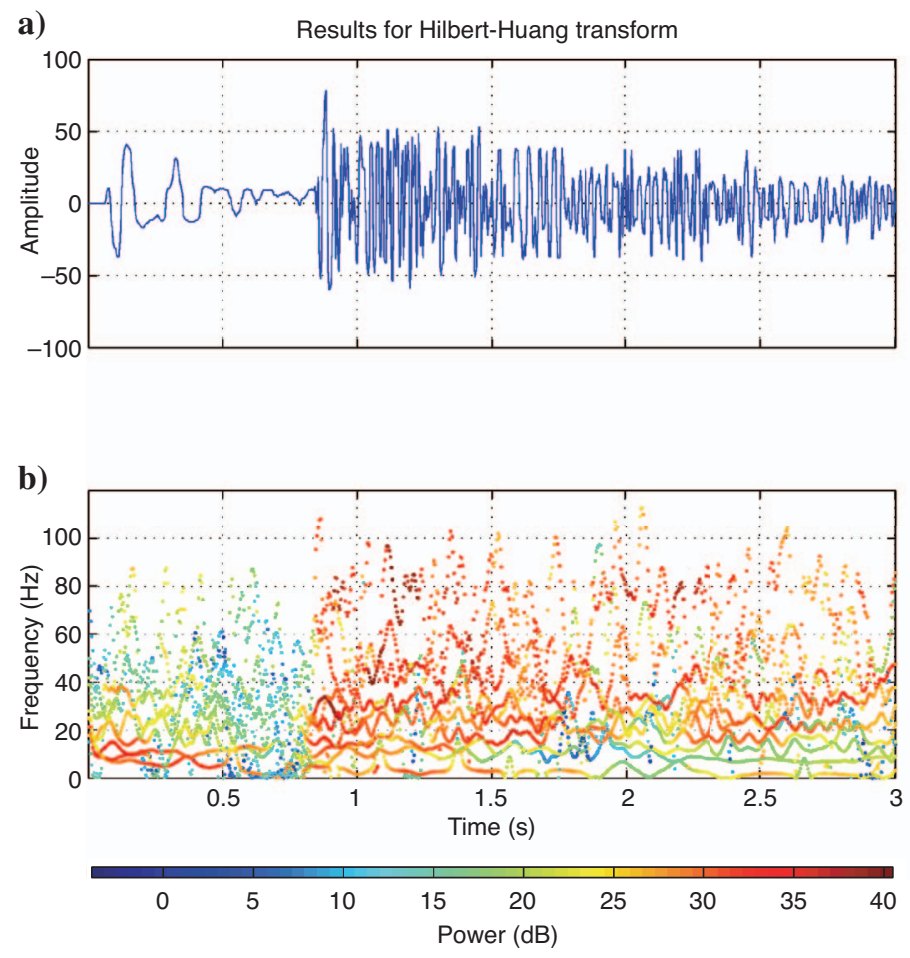

Figure 12. Hilbert transform of a stacked CDP gather prepared using EMD (i.e., HHT of a stacked CDP gather). (a) The time series analyzed. (b) Signal power plotted in time-frequency. (c) Hilbert marginal spectrum created as a temporal sum instantaneous amplitude for given frequencies. Notice how much information is now present in (b) compared to Figure 11.

forward and reverse filtering, and inherently remove more information than may be desired; resulting in amplitude decay and occasional phase changes as shown in Figure 9.

The use of EMD and HHT is a time-consuming process that may not be necessary for all data processing. This is the reason it is not presented in this work as a substitute for existing methods. Large targets, such as those typically addressed by the oil industry, may not require such advanced processing techniques. However, smaller targets, such as those in environmental geophysics, commonly demand such accuracy as the HHT can provide. The strength of the HHT for industry data lies in its ability to preserve phase and amplitude while empirically separating signal from noise. Geologic targets such as gas hydrates can be handled in the time-frequency domain by HHT with optimum results, meaning there is little noise introduced during processing. Additional success has been achieved removing ground roll from land seismics, as well as dewowing ground-penetrating radar data because both types of noise are very similar to the cablestrum example in this work. Future goals for this work involve integrating the HHT with amplitude-versus-offset processing of gas hydrates.

\section{CONCLUSIONS}

The EMD and HHT are significantly improved when data acquisition employs sample frequencies much higher than conventional Nyquist sampling; ten times faster than Nyquist seems to be a working minimum. This is referred to as oversampling, see Appendix A. The pros outweigh the cons in this situation. The cons are basically

c)

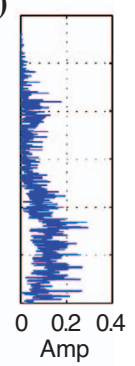

longer acquisition times and data storage problems. The pros are signals that represent geologic targets with very high resolution in both time and frequency. Additionally, the EMD, because of its iterative spline fitting, depends more on a signal's fidelity than on its frequency for analysis. Therefore, the HHT is also dependent on the signal's fidelity. In this manner, the accuracy of HHT filtering and the ability of it to optimally produce results is directly related to sample rate. It is intuitive that instantaneous attributes are only as instantaneous as the sample interval they are derived from. Therefore, high sample rates coupled with EMD and HHT allow for a wide range of tools to be developed.

\section{ACKNOWLEDGMENTS}

This work was funded by Minerals Management Service of the Department of Interior. This work is also a contribution to the Gulf of Mexico Hydrates Research Consortium which is supported by MMS, NETL of the Department of Energy, and NOAA of the Department of Commerce. The authors wish to thank Patrick Flandrin of the Laboratoire de Physique, École Normale Supérieure de Lyon for permission to modify and publish some of his MATLAB source codes used in this work.

\section{APPENDIX A}

\section{SAMPLING THEORY FOR SEISMIC PULSES}

Conventional sampling theory is based on a theorem of Shannon (1949) when he was working with the digitization of television signals. The sampling theorem relies on an assumption that the signal is limited in both time and frequency, "although it is not possible to fulfill both of these conditions exactly" (Shannon, 1949). The theorem states that a continuous-time signal can be digitized without loss of information by sampling it at a rate equal to one-half the highest frequency in its power spectrum. Shannon calls this the Nyquist frequency.

Of course, in the real world, all continuously recorded signals are of finite length. Slepian (1976) calls these real signals and discusses the problem of applying Shannon's sampling theorem to them. He shows that finite-length signals cannot be band-limited and, reciprocally, band-limited signals must be of infinite length with no beginning or end. The fact that Shannon was aware of this is indicated by his inclusion of the statement quoted above, that he felt no compulsion to discuss it further implies that it was of no great concern to his work. Though it does involve the time/bandwidth product, WT by both Shannon (1949) and Slepian (1976), which is well known to be subject to the Heisenberg Uncertainty Principle. In theory, the uncertainty associated with WT presents a serious dilemma when digitizing signals. Slepian (1976) suggests getting around it in practice by defining an insignificant level of power which would determine an effective bandwidth as signal power falls below it. 
Television signals consist of a high-frequency carrier that is modulated by subsignals which carry the information to be transmitted. The carrier-frequency portion of the signal is extremely long in time (an hour-long program is given as an example) and high in frequency relative to the subsignals. The application of the sampling theorem to such a signal would determine that a sampling rate equal to one-half the carrier frequency is sufficient. Given the great length of the carrier signal, this would be exact enough for the assumption to be approximately valid.

Seismic signals are causal and have finite energy; therefore, they are limited in time. However, they are not band-limited. This implies that they cannot be digitized at a finite rate without some loss of information. These losses take the form of quantization (round-off) errors caused by the finite dynamic range of the A-D converter and the aliasing of energy at frequencies too high for the chosen sampling rate to represent with good fidelity. Antialias filters are necessary but result in loss of information/resolution. A signal's capacity to transmit information is measured by its entropy, which is determined from its power spectrum (Shannon, 1948). Smoother power spectra exhibit greater entropy. The use of steep antialias filters disrupts the smoothness and lowers the entropy, thereby losing information. This can be avoided by sampling so fast that the Nyquist frequency is located far beyond any frequencies that contribute significantly to the signal's entropy. This allows the use of gentle antialias filters that are capable of reducing signal power enough to ensure that the $\mathrm{Ny}$ quist frequency represents an appropriate effective bandwidth. The technique has been dubbed oversampling and has found wide use in seismic and other applications requiring great resolution.

\section{REFERENCES}

Diaconescu, C., R. Kieckhefer, and J. Knapp, 2001, Geophysical evidence for gas hydrates in the deep water of the south Caspian Basin, Azerbaijan: Marine and Petroleum Geology, 18, 209-221.

Dix, H., 1949, On the minimum oscillatory character of spherical seismic pulses: Geophysics, 14, 17-20.

Flandrin, P., and P. Gonçalvès, 2004, Empirical mode decompositions as data-driven wavelet-like expansions for stochastic processes: International Journal of Wavelets, Multiresolution and Information Processes, 2, 477496.

Huang, N., Z. Shen, S. Long, M. Wu, E. Shih, Q. Zheng, C. Tung, and H. Liu, 1998, The empirical mode decomposition method and the Hilbert spectrum for non-stationary time series analysis: Proceedings of the Royal Society of London, A454, 903-995.

Magrin-Chagnolleau, I., and R. Baraniuk, 1999, Empirical mode decomposition based time-frequency attributes: 69th Annual International Meeting, SEG, Expanded Abstracts, 1949-1952.

McGee, T., 2000, Pushing the limits of high-resolution in marine seismic profiling: Journal of Environmental and Engineering Geophysics, 5, 43-53.

Oppenheim, A., and R. Schafer, 1989, Sampling of continuous-time signals, in Discrete-time signal processing: Prentice Hall, 879.

Rilling, G., P. Flandrin, and P. Gonçalvès, 2002, Empirical mode decomposition MATLAB codes: http://perso.ens-lyon.fr/patrick.flandrin/emd.html. , 2004a, Detrending and denoising with empirical mode decomposition: Eusipco, 12th European Signal Processing Conference.

, 2004b, Empirical mode decomposition as a filter bank: IEEE Signal Processing Letters, 11, 112-114.

Shannon, C., 1948, A mathematical theory of communication: The Bell System Technical Journal, 27, 379-423, 623-656.

- 1949, Communication in the presence of noise: Proceedings of the IRE, 37, 10-21.

Slepian, D., 1976, On bandwidth: Proceedings of the IEEE, 64, 292-300. 